\title{
Octonion Sparse Representation for Color and Multispectral Image Processing
}

\author{
Srđan Lazendić ${ }^{*}$, Hendrik De Bie* and Aleksandra Pižurica ${ }^{\dagger}$ \\ *Department of Mathematical Analysis, Clifford Research Group, \\ ${ }^{\dagger}$ Department of Telecommunications and Information Processing, UGent-IPI-imec \\ Faculty of Engineering and Architecture, Ghent University, Belgium \\ \{Srdan.Lazendic; Hendrik.DeBie; Aleksandra.Pizurica\}@UGent.be
}

\begin{abstract}
A recent trend in color image processing combines the quaternion algebra with dictionary learning methods. This paper aims to present a generalization of the quaternion dictionary learning method by using the octonion algebra. The octonion algebra combined with dictionary learning methods is well suited for representation of multispectral images with up to 7 color channels. Opposed to the classical dictionary learning techniques that treat multispectral images by concatenating spectral bands into a large monochrome image, we treat all the spectral bands simultaneously. Our approach leads to better preservation of color fidelity in true and false color images of the reconstructed multispectral image. To show the potential of the octonion based model, experiments are conducted for image reconstruction and denoising of color images as well as of extensively used Landsat 7 images.
\end{abstract}

Index Terms-Dictionary learning, Sparse representations, Octonions, Multispectral imaging, Landsat 7

\section{INTRODUCTION}

In the last two decades, the sparse representation model showed remarkable results for different image processing tasks. Many image and video processing problems benefit from the sparse representation model. The goal is to represent a given signal as a linear combination of only a few elements from the typically overcomplete set known as the dictionary. There are different ways of choosing a dictionary which best suits our needs. A first approach would be to use predesigned transform matrices such as overcomplete wavelets or curvelets, but although these dictionaries yield sparse representation of signals, dictionaries learned and trained on a set of representative examples are capable to give better results. The widely applied method for learning the dictionary is the so-called KSVD method [1]. Most dictionary learning methods as well as K-SVD, usually treat image patches as one-dimensional vectors, by stacking all the pixels into a single column vector, and then concatenating all the bands, regardless on the number of spectral channels that image has. In this way also multispectral and hyperspectral images are treated as large monochrome images so the interrelationship between the spectral channels is neglected.

Altough the quaternion algebra has been used for decades in computer graphics, robotics, signal representation and analysis [2], recently it was combined with standard dictionary learning techniques [3], [4]. More preciselly, since three imaginary units are well suited for representation of three color channels
( $R, G$ and $B$ ), the ideas of the Orthogonal Matching Pursuit method (OMP) [5], [6] for sparse coding as well as of K-SVD for dictionary training, were generalized to the quaternion setting in [3], [7] and are known as QOMP and K-QSVD. This approach showed extraordinary results in color image processing in terms of color fidelity, for different tasks such as image reconstruction, denoising, inpainting and super-resolution [3] as well as for color face recognition [4]. However, the existing quaternion-based approach is not applicable to images with more than three bands, e.g. multispectral images.

Motivated by the remarkable results obtained by the quaternion algebra for color image processing, here we extend the method of [3] with the usage of the 8-dimensional octonion algebra. The octonion algebra was developed for solving certain physical problems, and due to its property of non-associativity found its application in different fields such as exceptional Lie groups [8] and special relativity [9]. Application in analysis of analytic signals can be seen in [10] and a nice review of applications in physics can be found in [11]. The recovery of octonion signals from incomplete and noisy measurements has been addressed in [12]. To the best of our knowledge, our paper is the first one to report dictionary learning based on the octonion algebra.

Apart from finding the suitable algebraic structure that will generalize the quaternion model to a higher number of spectral bands, we aim at better preserving the spectral fidelity in the reconstructed image than the standard K-SVD model. The key idea in our approach is to represent multispectral image patches with 7 spectral channels as octonion signals. For more than 7 spectral channels, a dimensionality reduction technique can be applied which reduces the problem to a form for which our method is applicable. For a given set of training examples, where each example is an octonion signal, we learn the dictionary and find the sparse code in the form of octonion matrices by extending the standard Orthogonal Matching Pursuit (OMP) method [5], [6] and the Approximate K-SVD method [13] to the octonion setting. We name the method Octonion Dictionary Learning (ODL) and validate it for the reconstruction and the denoising of color and Landsat 7 satellite images.

The organization of the paper is as follows. In Section II we give the preliminaries about the octonion algebra which will be useful in order to derive the octonion sparse representation. 
This follows in Section III together with the problem formulation. Section IV introduces the main novelties in our ODL model. In Section $\mathrm{V}$ we present experimental results which were validated on color and Landsat 7 images. Section VI concludes the paper.

\section{PREliminaries}

\section{A. Algebra of octonions}

The octonions are an 8-dimensional unital, distributive algebra over $\mathbb{R}$ with basis $\left\{1, e_{1}, e_{2}, e_{3}, e_{4}, e_{5}, e_{6}, e_{7}\right\}$ where 1 is the multiplicative identity and $e_{i}, i \in\{1, \ldots, 7\}$ are imaginary units that satisfy $e_{i}^{2}=-1$, with the multiplication rules between the basis elements given in Table I.

TABLE I

MULTIPLICATION RULES BETWEEN BASIS ELEMENTS.

\begin{tabular}{r|rrrrrrrr} 
& 1 & $e_{1}$ & $e_{2}$ & $e_{3}$ & $e_{4}$ & $e_{5}$ & $e_{6}$ & $e_{7}$ \\
\hline 1 & 1 & $e_{1}$ & $e_{2}$ & $e_{3}$ & $e_{4}$ & $e_{5}$ & $e_{6}$ & $e_{7}$ \\
$e_{1}$ & $e_{1}$ & -1 & $e_{3}$ & $-e_{2}$ & $e_{5}$ & $-e_{4}$ & $-e_{7}$ & $e_{6}$ \\
$e_{2}$ & $e_{2}$ & $-e_{3}$ & -1 & $e_{1}$ & $e_{6}$ & $e_{7}$ & $-e_{4}$ & $-e_{5}$ \\
$e_{3}$ & $e_{3}$ & $e_{2}$ & $-e_{1}$ & -1 & $e_{7}$ & $-e_{6}$ & $e_{5}$ & $-e_{4}$ \\
$e_{4}$ & $e_{4}$ & $-e_{5}$ & $-e_{6}$ & $-e_{7}$ & -1 & $e_{1}$ & $e_{2}$ & $e_{3}$ \\
$e_{5}$ & $e_{5}$ & $e_{4}$ & $-e_{7}$ & $e_{6}$ & $-e_{1}$ & -1 & $-e_{3}$ & $e_{2}$ \\
$e_{6}$ & $e_{6}$ & $e_{7}$ & $e_{4}$ & $-e_{5}$ & $-e_{2}$ & $e_{3}$ & -1 & $-e_{1}$ \\
$e_{7}$ & $e_{7}$ & $-e_{6}$ & $e_{5}$ & $e_{4}$ & $-e_{3}$ & $-e_{2}$ & $e_{1}$ & -1
\end{tabular}

In order to introduce an octonion sparse model we will need the following algebraic properties of the octonion algebra. If $x \in \mathbb{O}$ is an octonion then it can be written as

$$
x=x_{0}+x_{1} e_{1}+\cdots+x_{7} e_{7}=x_{0}+\sum_{i=1}^{7} x_{i} e_{i}
$$

where each $x_{i} \in \mathbb{R}$. The conjugate of an octonion is defined as $\bar{x}=x_{0}-\sum_{i=0}^{7} x_{i} e_{i}$ and the norm is introduced as

$$
|x|=\sqrt{x \bar{x}}=\sqrt{\bar{x} x}=\sqrt{x_{0}^{2}+\cdots+x_{7}^{2}} .
$$

The octonions are also a division and a composition algebra which means that $|x y|=|x||y|$ holds for all $x, y \in \mathbb{O}$. It is important to stress that the octonion algebra is noncommutative and non-associative. This means that for arbitrary $x, y, z \in \mathbb{O}: \quad x y \neq y x$ and $x(y z) \neq(x y) z$. However, in general it holds that $x \bar{x}=\bar{x} x$, for every $x \in \mathbb{O}$. More about octonions and their applications can be found in [11].

\section{B. Octonion vectors and matrices}

To treat multispectral images with up to 7 spectral channels we will need to introduce octonion vectors and octonion matrices. The non-associativity complicates vector analysis over $\mathbb{O}$, as we will see in the sequel.

The octonion vector $\mathbf{x} \in \mathbb{O}^{n \times 1}$ is a vector where each entry is an octonion. Often it will be useful to write it as

$$
\mathbf{x}=\mathbf{x}_{0}+\mathbf{x}_{1} e_{1}+\ldots \mathbf{x}_{7} e_{7}
$$

where now each vector $\mathbf{x}_{i} \in \mathbb{R}^{n \times 1}$ for $i=1, \ldots, n$. Similarly, the octonion matrix $\mathbf{A}=\left[a_{i j}\right]_{i, j=1}^{m, n} \in \mathbb{O}^{m \times n}$ is a matrix with entries in $\mathbb{O}$. Also, we can write it as

$$
\mathbf{A}=\mathbf{A}_{0}+\mathbf{A}_{1} e_{1}+\cdots+\mathbf{A}_{7} e_{7},
$$

where each $\mathbf{A}_{i} \in \mathbb{R}^{m \times n}$, for $i=0, \ldots, 7$.

The conjugation can be extended to vectors and matrices. The conjugate transpose matrix of a matrix $\mathbf{A}=\left[a_{i j}\right]_{i, j=1}^{m, n} \in$ (1) $^{m \times n}$ is defined as

$$
\mathbf{A}^{*}=\left[\overline{a_{j i}}\right]_{j, i=1}^{n, m} \in \mathbb{O}^{n \times m} .
$$

The inner product can be introduced as a function $\langle\cdot, \cdot\rangle: \mathbb{O}^{n} \times$ $\mathbb{O}^{n} \rightarrow \mathbb{O}$ given by $\langle\mathbf{x}, \mathbf{y}\rangle=\mathbf{x}^{*} \mathbf{y}=\sum_{i=1}^{n} \overline{x_{i}} y_{i}$. Then, for an octonion vector $\mathbf{x} \in \mathbb{O}^{n \times 1}$ we can define its norm by $\|\mathbf{x}\|_{2}=\sqrt{\mathbf{x}^{*} \mathbf{x}}=\sqrt{\sum_{i=1}^{n}\left|x_{i}\right|^{2}}$ where $x_{i}, i=1, \ldots, n$ are the entries of the vector $\mathbf{x}$. The Frobenius norm of an octonion matrix $\mathbf{A}=\left[a_{i j}\right]$ is given by $\|\mathbf{A}\|_{F}=\sqrt{\sum_{i=1}^{m} \sum_{j=1}^{n}\left|a_{i j}\right|^{2}}$.

Since octonions are non-associative there is no real matrix representation that can faithfully represent them. However, we can use the so-called pseudo-real matrix representation as introduced in [14], [15] which is suitable for our work because it represents matrix-vector multiplication. If $x=$ $x_{0}+\sum_{i=1}^{7} x_{i} e_{i} \in \mathbb{O}$ is an octonion then we define the realvector representation $\nu(x) \in \mathbb{R}^{8 \times 1}$ as $\nu(x)=\left[\begin{array}{llll}x_{0} & x_{1} & \ldots & x_{7}\end{array}\right]^{T}$. In this way the real-linear isomorphism $\nu: \mathbb{O} \rightarrow \mathbb{R}^{8 \times 1}$ is obtained. Similarly, we can define matrices $C_{j} \in \mathbb{R}^{8 \times 8}$ for $j=1, \ldots, 7$ by the property

$$
C_{j} \nu\left(e_{k}\right)=\nu\left(e_{j} e_{k}\right), \quad k=0, \ldots, 7 .
$$

Then from Table I we can easily conclude that for every $j=$ $1, \ldots, 7$ there holds $C_{j}^{2}=-I$, where $I$ is the identity matrix. Then the real linear, injective map $\chi: \mathbb{O} \rightarrow \mathbb{R}^{8 \times 8}$ can be defined as

$$
\chi\left(\sum_{i=1}^{7} x_{i} e_{i}\right)=x_{0} I+\sum_{i=1}^{7} x_{i} C_{i} .
$$

After expanding the expression on the right in (5) we obtain a matrix with columns that are mutually orthogonal vectors.

The same maps can be extended to octonion vectors and matrices. If we use the same notation for maps $\nu$ and $\chi$ that act on entries of the vectors and matrices, respectively, then we are able to define multiplication between octonion vectors and octonion matrices by using the real matrix multiplication. Indeed, for every $\mathbf{A} \in \mathbb{O}^{m \times n}$ and every $\mathbf{x} \in \mathbb{O}^{n \times 1}$ it holds that

$$
\nu(\mathbf{A x})=\chi(\mathbf{A}) \nu(\mathbf{x}) .
$$

Note that for $\mathbf{x} \in \mathbb{O}^{n \times 1}$ it holds

$$
\|\mathbf{x}\|_{2}=\|\nu(\mathbf{x})\|_{2}
$$

The proofs of all the matrix representations presented here can be found in [14], [15].

\section{PROBLEM FORMULATION}

\section{A. Octonion representation of multispectral images}

Let $\mathbf{y}$ be an $\sqrt{m} \times \sqrt{m}$ image patch extracted from a multispectral image with up to 7 channels which is then transformed into an octonion column vector of size $m \times 1$. The octonion algebra is well suited for the representation of this image patch since every spectral channel can be assigned 
to one of the seven imaginary units $e_{1}, \ldots, e_{7}$. This image patch is then treated as an octonion vector $\mathbf{y} \in \mathbb{O}^{m \times 1}$ which can be represented as

$$
\mathbf{y}=0+\mathbf{y}_{1} e_{1}+\cdots+\mathbf{y}_{7} e_{7}, \quad \mathbf{y}_{i} \in \mathbb{R}^{m \times 1},
$$

so every channel of this patch is the real vector $\mathbf{y}_{i} \in \mathbb{R}^{m \times 1}$. Note that this representation is a generalization of the quaternion representation model introduced in [3], [7] that was used for RGB color images. Indeed, if $\mathbf{y}_{4}, \ldots, \mathbf{y}_{7}$ are zero vectors then we have only three imaginary units which represent, e.g. $\mathrm{R}, \mathrm{G}$ and $\mathrm{B}$ color channels.

\section{B. Sparse coding and dictionary learning problems}

The goal of octonion sparse coding is to represent a given octonion signal i.e. a signal given as an octonion vector $\mathbf{y} \in$ $\mathbb{O}^{m \times 1}$ by the linear combination of only a few elements from a redundant (overcomplete) set $\mathbf{D}=\left\{\mathbf{d}_{k}\right\}_{k=1}^{m} \in \mathbb{O}^{m \times n}$ which is called a dictionary and whose columns $\mathbf{d}_{k}$ are unit norm vectors known as atoms. Thus if the dictionary $\mathbf{D}$ is given, the aim is to find the $L$-sparse vector of coefficients $\mathbf{x} \in \mathbb{O}^{n \times 1}$ known as the sparse code such that $\|\mathbf{x}\|_{0} \leq L$ and

$$
\mathbf{y} \approx \mathbf{D x}
$$

where $L=$ const. is the prescribed number of non-zero octonion elements in the octonion vector $\mathbf{x}$, i.e. the sparsity level. The pseudo-norm $\ell_{0}$ just counts the number of these non-zero elements of the given vector and the goal is to solve the sparse coding problem

$$
\hat{\mathbf{x}}=\underset{\mathbf{x}}{\arg \min }\|\mathbf{y}-\mathbf{D} \mathbf{x}\|_{2}^{2} \quad \text { s.t. } \quad\|\mathbf{x}\|_{0} \leq L .
$$

If we are provided with a set of training samples i.e. the set of octonionic signals $\mathbf{Y}=\left\{\mathbf{y}_{k}\right\}_{k=1}^{p} \in \mathbb{O}^{m \times p}$, then the dictionary learning problem consists of finding the dictionary $\mathbf{D} \in \mathbb{O}^{m \times n}$, which best adapts to the given training set $\mathbf{Y}$, and the sparse code $\mathbf{X}=\left\{\mathbf{x}_{k}\right\}_{k=1}^{p} \in \mathbb{O}^{n \times p}$ such that $\mathbf{Y} \approx \mathbf{D X}$. The dictionary learning problem can be formulated as the following minimization problem

$$
\begin{array}{r}
\{\hat{\mathbf{D}}, \hat{\mathbf{X}}\}=\underset{\mathbf{D}, \mathbf{X}}{\arg \min }\left\{\|\mathbf{Y}-\mathbf{D} \mathbf{X}\|_{F}^{2}\right\} \\
\text { s.t. } \quad\left\|\mathbf{x}_{k}\right\|_{0} \leq L, \quad k=1, \ldots, p .
\end{array}
$$

\section{PRoposed METHOD}

\section{A. Sparse coding step}

The problem formulated in (10) can be seen as a generalization of the complex and quaternion sparse coding problems [16]. Indeed, in the expanded form we see that the coefficient matrix after multiplication is given as in (5) and that the columns of the coefficient matrix are orthogonal. So as in the quaternion model we obtain a structured coefficient matrix which preserves the correlation between spectral channels and the orthogonality property. Linear correlation among the color channels, which has proven to be useful for the preservation of color fidelity, remains valid. In this way the relationship among the image channels is much better preserved than in the standard concatenation model or in the band-wise K-SVD model for multichannel images. In [12], an algorithm has been introduced for solving (10) by solving the equivalent $\ell_{1}$-norm minimization problem for octonionic signals by converting it into an equivalent problem over $\mathbb{R}$, which is then solved as a real convex optimization problem.

Here we introduce a new algorithm for solving (10) based on the idea of the Orthogonal Matching Pursuit (OMP) [5] but adapted to the octonion settings. Actually, in each step $k$ the algorithm selects the atom $\mathbf{d}_{k}$ that produces the strongest decrease of the residual $\left\|\mathbf{r}^{k}\right\|_{2}^{2}=\left\|\mathbf{r}^{k-1}-\mathbf{d}_{k} \mathbf{x}_{k}\right\|_{2}^{2}$, with $\mathbf{r}^{0}=\mathbf{y}$. It can be shown that in the octonion setting this is equivalent with selecting the atom that is most correlated with the residual vector i.e. $\left\langle\mathbf{r}^{k}, \mathbf{d}_{k}\right\rangle$. Due to space limitation the proof is omitted here and will follow in the future work. After choosing the atom that produces the maximum absolute value in the inner product with the residual, the active dictionary is formed $\mathbf{D}^{k}=\left[\mathbf{D}^{k-1}, \mathbf{d}_{k}\right]$ and the coding coefficients $\mathbf{x}$ are selected so that the norm $\left\|\mathbf{y}-\mathbf{D}^{k} \mathbf{x}\right\|_{2}^{2}$ is minimized. In the complex and quaternion settings the problem of minimization of $\left\|\mathbf{y}-\mathbf{D}^{k} \mathbf{x}\right\|_{2}^{2}$ has a closed form solution which is given as a multiplication of pseudo-inverse of the dictionary matrix with the signal vector (see [16]). Due to difficulties of solving linear least square problem in the octonion setting we use a different strategy by transforming this minimization problem into a real vector minimization problem by using (7) and (6). Indeed, since

$$
\begin{aligned}
\left\|\mathbf{y}-\mathbf{D}^{k} \mathbf{x}\right\|_{2}^{2} & =\left\|\nu\left(\mathbf{y}-\mathbf{D}^{k} \mathbf{x}\right)\right\|_{2}^{2} \\
& =\left\|\nu(\mathbf{y})-\chi\left(\mathbf{D}^{k}\right) \nu(\mathbf{x})\right\|_{2}^{2},
\end{aligned}
$$

by minimizing the last expression on the right in (12) we can easily solve the real minimization problem and then obtain the octonion coefficient vector $\mathbf{x}$ by using the inverse map $\nu^{-1}$.

\section{B. Dictionary update step}

In order to solve the dictionary learning problem in (11) we will iterate between two steps: sparse coding step (which is performed by the proposed octonion OMP) and dictionary update step. The algorithm presented here is based on the idea of the Approximate K-SVD [13]. Due to the inability to find eigenvectors and eigenvalues of octonion matrices (so far this is solved only for $2 \times 2$ and $3 \times 3$ octonion Hermitian matrices - e.g. [17]) which results in the inability of defining inverses and pseudoinverses of octonion matrices, we cannot use the classical K-SVD model. But, as it was stressed in [13], the exact calculation of the inverse matrix is not really a necessity.

Indeed, the previous idea can be extended to the octonion setting and in this way we are able to solve the one-rank minimization problem without exact computation of the SVD decomposition of octonion matrices. Namely, the dictionary update stage can be performed via alternating minimization, i.e. by fixing one variable and solving the minimization problem with respect to the second variable. Exact calculations with proofs that in the octonion setting this indeed leads to a solution of the minimization problem in (11) are space consuming and will not be presented here. 


\section{EXPERIMENTS AND VALIDATION}

We demonstrate the potential of our method by illustrating its applications in reconstruction and denoising of multichannel images. All dictionaries were trained in 10 iterations, on the same set of images of size $512 \times 512$. The sparsity level $L \leq 5$ for ODL and K-QSVD was used, and in order to ensure fair comparison, for concatenated K-SVD model we use sparsities $L \leq 20$ and $L \leq 40$ for color and Landsat 7 images, respectively. ${ }^{1}$ The same sparsity levels were used later for image reconstruction. The dataset of training samples, both for the color and for the Landsat 7 image processing, consists of 10000 fully overlapping image patches of size $8 \times 8$, but we can also choose patches of different size $($ e.g. $5 \times 5$ ). The experiments for image reconstruction from complete data were repeated for different sizes of dictionary, but for denoising we always use a dictionary of size $64 \times 256$.

Now we will formulate the image denoising problem that we will solve in the sequel by using the proposed ODL model. Let $\mathbf{Y} \in \mathbb{O} \sqrt{m} \times \sqrt{m}$ be an ideal noise-free image and let $\mathbf{Z}$ be its noisy version $\mathbf{Z}=\mathbf{Y}+\mathbf{w}$, with $\mathbf{w}$ being additive white Gaussian noise with standard deviation $\sigma$. Assuming that all the image patches from $\mathbf{Y}$ admit a sparse representation, we want to solve the following minimization problem

$$
\begin{array}{r}
\left\{\hat{\mathbf{D}}, \hat{\mathbf{x}}_{i j}, \hat{\mathbf{Y}}\right\}=\underset{\mathbf{D}, \mathbf{x}_{i j}, \mathbf{Y}}{\arg \min }\left\{\lambda\|\mathbf{Z}-\mathbf{Y}\|_{2}^{2}+\right. \\
\left.\sum_{i j} \mu_{i j}\left\|\mathbf{x}_{i j}\right\|_{0}+\sum_{i j}\left\|\mathbf{D} \mathbf{x}_{i j}-R_{i j} \mathbf{Y}\right\|_{2}^{2}\right\},
\end{array}
$$

where $\hat{\mathbf{Y}}$ is the estimator of $\mathbf{Y}$ and $R_{i j} \mathbf{Y}$ extracts a $\sqrt{m} \times \sqrt{m}$ patch at position $(i, j)$ from $\mathbf{Y}$. Further, $\hat{\mathbf{x}}_{i j}$ represents the coefficient vector for the patch located at position $(i, j)$ and $\hat{\mathbf{D}}$ is the estimation of the optimal dictionary which leads to the sparsest representation of the recovered image. During the computation of the representation vectors $\mathbf{x}_{i j}$ for each image patch $R_{i j} \mathbf{Y}$, the octonion OMP refers to

$$
\underset{\mathbf{x}_{i j}}{\arg \min }\left\|\mathbf{x}_{i j}\right\|_{0} \quad \text { s.t. }\left\|\mathbf{D} \mathbf{x}_{i j}-R_{i j} \mathbf{Y}\right\|_{2}^{2} \leq n(C \sigma)^{2},
$$

with $\sigma$ being the standard deviation of the Gaussian noise. The image is then reconstructed by simple averaging of the denoised patches. Empirically, in our method we set $C=1.15$ and $\lambda=0.04$.

\section{A. Color image denoising}

The proposed octonion dictionary learning model as an extension of K-QSVD towards more channels is applicable to standard color images as well, where the three color channels are assigned to 3 out of 7 imaginary units. In order to verify this experimentaly the ODL method was compared with $\mathrm{K}$ QSVD [3] and the traditional K-SVD model (introduced in [18]) in the task of color image denoising. Figure 1 shows image denoising results for two test images. In both cases,

\footnotetext{
${ }^{1}$ In the ODL model, every element in the coefficient vector is an octonion, so each of them has 8 values in itself. The ratio for the comparison with the K-SVD model is $1: 8$ so the same number of non-zero elements in the coefficient matrix $\mathbf{X}$ is used.
}

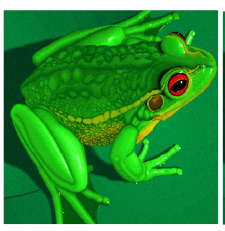

(a) $\sigma=10$

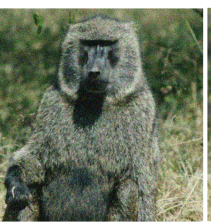

(e) $\sigma=25$

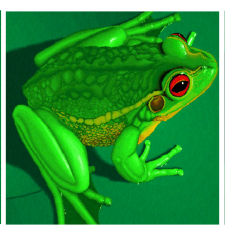

(b) $33.53 \mathrm{~dB}$

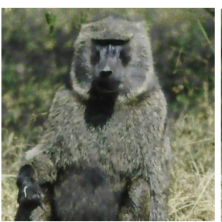

(f) $29.28 \mathrm{~dB}$ (c) $34.42 \mathrm{~dB}$

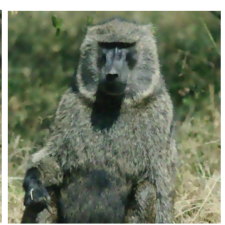

(g) $30.43 \mathrm{~dB}$

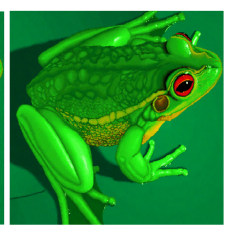

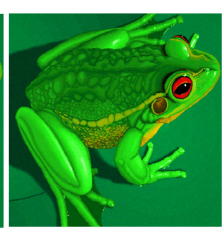

(d) $35.13 \mathrm{~dB}$

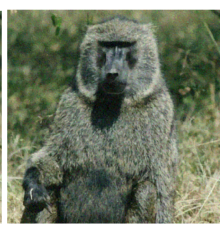

(h) $30.45 \mathrm{~dB}$
Fig. 1. Color image denoising. First column - noisy images with Gaussian noise. Second column - K-SVD denoising. Third column - K-QSVD denoising. Fourth column - ODL denoising. PSNR values are below the images.

K-QSVD and ODL improve upon the traditional K-SVD. The proposed ODL yielded a better result than K-QSVD on the less noisy image and a similar result on the noisier one. ODL also demonstrated somewhat better texture preservation at the expense of some residual noise.

TABLE II

DENOISING OF COLOR IMAGES WITH AVERAGE VALUES OF PSNR/SSIM OBTAINED OVER THE SET OF TEST IMAGES

\begin{tabular}{|c|c|c|c|}
\cline { 2 - 4 } \multicolumn{1}{c|}{} & \multicolumn{3}{c|}{ Average values of PSNR/SSIM } \\
\hline & K-SVD & K-QSVD & ODL \\
\hline$\sigma=10$ & $34.40 \mathrm{~dB} / 0.966$ & $34.53 \mathrm{~dB} / 0.902$ & $\mathbf{3 5 . 3 2 d B / 0 . 9 7 1}$ \\
\hline$\sigma=25$ & $29.00 \mathrm{~dB} / 0.880$ & $\mathbf{3 0 . 6 5} \mathrm{dB} / 0.819$ & $29.94 \mathrm{~dB} / \mathbf{0 . 8 8 9}$ \\
\hline
\end{tabular}

Average values of two metrics: peak signal to noise ratio (PSNR) and structural similarity index (SSIM), obtained on a set of five test images (frog, safari16, Lena, peppers and pelican) are reported in Table II. The results show that at relatively small noise levels our method outperforms the other two dictionary learning methods in both PSNR and SSIM values. At higher noise levels, the proposed ODL still outperforms K-SVD and yields similar results as K-QSVD, while being applicable to images with more than three channels in contrast to K-QSVD.

\section{B. Denoising Landsat 7 data}

In this Section we validate the proposed ODL method on images with more than three channels. In particular, we use two multi-spectral remote sensing images acquired by Landsat 7: Montana and Mississipi (which are included in Matlab, see [19]). The main motivation behind our method with higherdimensional algebraic structure is the preservation of spectral fidelity after data processing. Since K-QSVD is not applicable to images with more than three channels, we compare the results to K-SVD only. In a first experiment, we evaluate image reconstruction quality from complete data. The average reconstruction PSNR/SSIM values for the two tested images are $42.47 \mathrm{~dB} / 0.9978$ and $40.90 \mathrm{~dB} / 0.9960$ for $\mathrm{ODL}$ and $\mathrm{K}$ SVD, respectively. Table III reports image denoising results for 


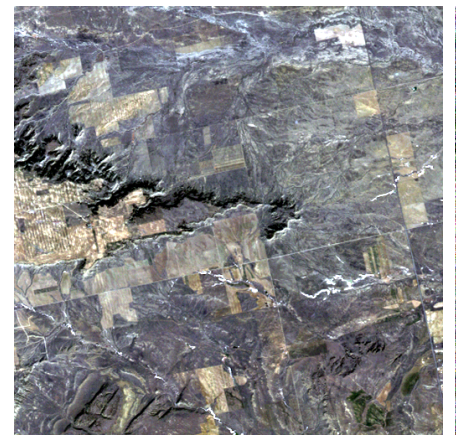

(a) Original noise-free image

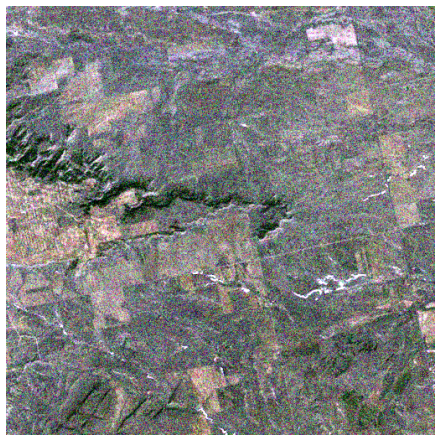

(b) $28.13 \mathrm{~dB} / 0.5068$

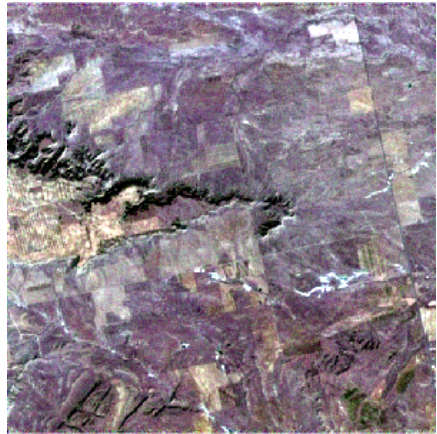

(c) $34.79 \mathrm{~dB} / 0.991$

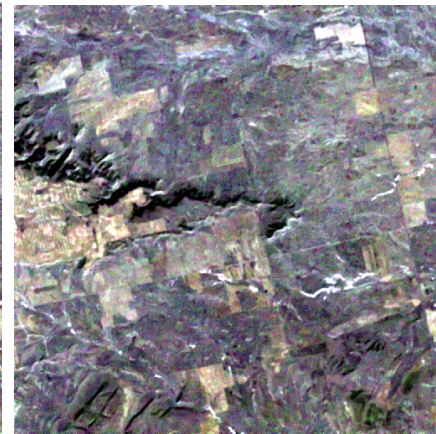

(d) $36.79 \mathrm{~dB} / 0.9000$

Fig. 2. Noisy Montana image (b) with $\sigma=10$ denoised by the K-SVD method (c) and the proposed ODL method (d) with average PSNR/SSIM values.

different levels of additive white Gaussian noise. Experiments were repeated 10 times for both images and the average value for each image is showed. Note that our method gives better results for $\sigma=10$ and in Figure 2 the color fidelity in the reconstructed true color image is better preserved than with the K-SVD model. Also, for higher levels of Gaussian noise the algorithm tends to give more or less similar results as KSVD (see Table III).

TABLE III

DENOISING OF LANDSAT 7 IMAGES WITH AVERAGE PSNR/SSIM VALUES AFTER REPETITION OF EXPERIMENTS ON THE SPECIFIED IMAGES

\begin{tabular}{|c|c|c|c|c|}
\cline { 2 - 5 } \multicolumn{1}{c|}{} & \multicolumn{2}{c|}{ Montana image } & \multicolumn{2}{c|}{ Mississippi image } \\
\hline & K-SVD & ODL & K-SVD & ODL \\
\hline$\sigma=10$ & $34.79 / \mathbf{0 . 9 9 1}$ & $\mathbf{3 6 . 7 9} / 0.900$ & $36.21 / \mathbf{0 . 9 8 9}$ & $\mathbf{3 8 . 7 1} / 0.918$ \\
\hline$\sigma=25$ & $31.63 / \mathbf{0 . 9 8 1}$ & $\mathbf{3 2 . 3 0} / 0.765$ & $32.54 / \mathbf{0 . 9 6 9}$ & $\mathbf{3 3 . 0 9} / 0.772$ \\
\hline Average & $33.21 / \mathbf{0 . 9 8 6}$ & $\mathbf{3 4 . 5 4} / 0.832$ & $34.37 / \mathbf{0 . 9 7 9}$ & $\mathbf{3 5 . 9 0} / 0.845$ \\
\hline
\end{tabular}

The test platform was MATLAB on Intel Core-i7 CPU with 16GB memory. The average time for the reconstruction from the complete data is 350 seconds for the ODL model and 240 seconds for the K-SVD model. Similarly, for the denoising the approximate time is 800 seconds for ODL and 600 seconds for K-SVD.

\section{CONCLUSION}

In this paper, we proposed a new model which can be applied for image processing tasks such as image reconstruction and image denoising of multichannel images. We combine the octonion algebra with dictionary learning techniques. Also the new algorithm for solving the octonion sparse coding problem, named as octonion orthogonal matching pursuit as well as the octonion dictionary learning (ODL) technique, based on the alternating minimization technique, were introduced. The experiments conducted for the reconstruction and denoising of color and Landsat 7 satellite images showed improvements in PSNR values in the reconstruction compared to the traditional concatenated K-SVD model.

\section{ACKNOWLEDGMENT}

This research has been partially supported by the institutional grant no. BOF15/24J/078 of Ghent University.

\section{REFERENCES}

[1] M. Aharon, M. Elad, and A. Bruckstein, "K-SVD: An algorithm for designing overcomplete dictionaries for sparse representation," IEEE Transactions on signal processing, vol. 54, no. 11, pp. 4311-4322, 2006.

[2] T. A. Ell, N. Le Bihan, and S. J. Sangwine, Quaternion Fourier transforms for signal and image processing. John Wiley \& Sons, 2014.

[3] Y. Xu, L. Yu, H. Xu, H. Zhang, and T. Nguyen, "Vector sparse representation of color image using quaternion matrix analysis," IEEE Transactions on image processing, vol. 24, no. 4, pp. 1315-1329, 2015.

[4] C. Zou, K. I. Kou, and Y. Wang, "Quaternion collaborative and sparse representation with application to color face recognition," IEEE Transactions on image processing, vol. 25, no. 7, pp. 3287-3302, 2016.

[5] S. G. Mallat and Z. Zhang, "Matching pursuits with time-frequency dictionaries," IEEE Transactions on signal processing, vol. 41, no. 12, pp. 3397-3415, 1993.

[6] Y. C. Pati, R. Rezaiifar, and P. S. Krishnaprasad, "Orthogonal matching pursuit: Recursive function approximation with applications to wavelet decomposition," in 1993 Conference Record of The Twenty-Seventh Asilomar Conference on Signals, Systems and Computers. IEEE, 1993, pp. 40-44.

[7] L. Yu, Y. Xu, H. Xu, and H. Zhang, "Quaternion-based sparse representation of color image," in 2013 International Conference on Multimedia and Expo (ICME). IEEE, 2013, pp. 1-7.

[8] J. C. Baez and J. Huerta, " $G_{2}$ and the rolling ball," Trans. Amer. Math. Soc., vol. 366, no. 10, pp. 5257-5293, 2014.

[9] J. Baez, "The octonions," Bulletin of the American Mathematical Society, vol. 39, no. 2, pp. 145-205, 2002.

[10] S. L. Hahn and K. M. Snopek, Complex and Hypercomplex Analytic Signals: Theory and Applications. Artech House, 2016.

[11] T. Dray and C. A. Manogue, The geometry of the octonions. World Scientific, 2015.

[12] R. Wang, G. Xiang, and F. Zhang, " $L_{1}$-norm minimization for octonion signals," in 2016 International Conference on Audio, Language and Image Processing (ICALIP). IEEE, 2016, pp. 552-556.

[13] R. Rubinstein, M. Zibulevsky, and M. Elad, "Efficient implementation of the K-SVD algorithm using batch orthogonal matching pursuit," $C S$ Technion, vol. 40, no. 8, pp. 1-15, 2008

[14] L. Rodman, "Hermitian octonion matrices and numerical ranges," Electronic Journal of Linear Algebra, vol. 27, no. 1, p. 33, 2014.

[15] Y. Tian, "Matrix representations of octonions and their applications," Advances in Applied Clifford Algebras, vol. 10, no. 1, pp. 61-90, 2000.

[16] Q. Barthélemy, A. Larue, and J. I. Mars, "Sparse approximations for quaternionic signals," Advances in Applied Clifford Algebras, vol. 24, no. 2, pp. 383-402, 2014.

[17] T. Dray and C. A. Manogue, "The octonionic eigenvalue problem," Advances in Applied Clifford Algebras, vol. 8, no. 2, pp. 341-364, 1998.

[18] J. Mairal, M. Elad, and G. Sapiro, "Sparse representation for color image restoration," IEEE Transactions on image processing, vol. 17, no. 1, pp. 53-69, 2008.

[19] G. Siogkas, Visual Media Processing Using Matlab Beginner's Guide. Packt Publishing Ltd, 2013. 